\title{
Early Type Galaxies in the Mid Infrared: a new flavor to their stellar populations $\dagger$
}

\author{
A. Bressan ${ }^{1,2,3}$, P. Panuzzo ${ }^{1}$, O. Vega ${ }^{3}$, L. Buson ${ }^{1}$, M. Clemens ${ }^{1}$, \\ G.L. Granato ${ }^{1,2}$, R. Rampazzo ${ }^{1}$, L. Silva ${ }^{4}$ and J.R. Valdes ${ }^{3}$ \\ ${ }^{1}$ INAF Osservatorio Astronomico di Padova, vicolo dell'Osservatorio 5, 35122 Padova, Italy \\ email: alessandro.bressan@oapd.inaf.it \\ ${ }^{2}$ SISSA, via Beirut 4, 34014, Trieste, Italy \\ ${ }^{3}$ INAOE, Luis Enrique Erro 1, 72840, Tonantzintla, Puebla, Mexico \\ ${ }^{4}$ INAF Osservatorio Astronomico di Trieste, Via Tiepolo 11, I-34131 Trieste, Italy
}

\begin{abstract}
The mid infrared emission of early type galaxies traces the presence of intermediate age stellar populations as well as even tiny amounts of ongoing star formation. Here we discuss high S/N Spitzer IRS spectra of a sample of Virgo early type galaxies, with particular reference to NGC 4435. We show that, by combining mid infrared spectroscopic observations with existing broad band fluxes, it is possible to obtain a very clean picture of the nuclear activity in this galaxy.
\end{abstract}

Keywords. galaxies: elliptical and lenticular, cD, galaxies: ISM, infrared: galaxies

\section{Introduction}

With the advent of the Spitzer Space Telescope new frontiers have been opened in the study of stellar population content of early-type galaxies (ETGs) and, in particular, the ability to quantify the occurrence and strength of the rejuvenation episodes. By means of mid infrared (MIR) observations it is possible to detect the presence of intermediate age stellar populations in passively evolving galaxies, and measure even tiny amounts of ongoing star formation activity.

Bressan, Granato \& Silva (1998) suggested that the MIR spectral region of old and intermediate age stellar populations should be affected by the presence of mass-losing oxigen-rich AGB giants. Their integrated emission around $10 \mu \mathrm{m}$ should be clearly seen in passively evolving galaxies; its analysis, in combination with UV, optical and NIR observations, should provide accurate age-metallicity ranking, unbiased by the age-metallicity degeneracy.

Moreover, ongoing star formation can be easily detected in the MIR, from the presence of prominent emission features such as PAHs and atomic or molecular emission lines (e.g. Kaneda et al. 2005, Bressan et al. 2006a,b, Panuzzo et al. 2007). Last but not least, MIR nebular lines constitute a strong diagnostic to disentangle star formation and AGN activity and they also allow a direct and perhaps unique determination of the chemical abundance of the surrounding gas (Panuzzo et al. 2007).

For the above reasons we begun a systematic study of the properties of ETGs in the mid infrared spectral region with the Spitzer Space Telescope. Here we report on the results obtained with Spitzer IRS (Houck et al. 2004) MIR spectroscopic observations of a sample of ETGs in the Virgo cluster (Bressan et al 2006a,b; Bressan et al 2007).

$\dagger$ This work is based on observations made with the Spitzer Space Telescope, which is operated by the JPL, Caltech under a contract with NASA. 

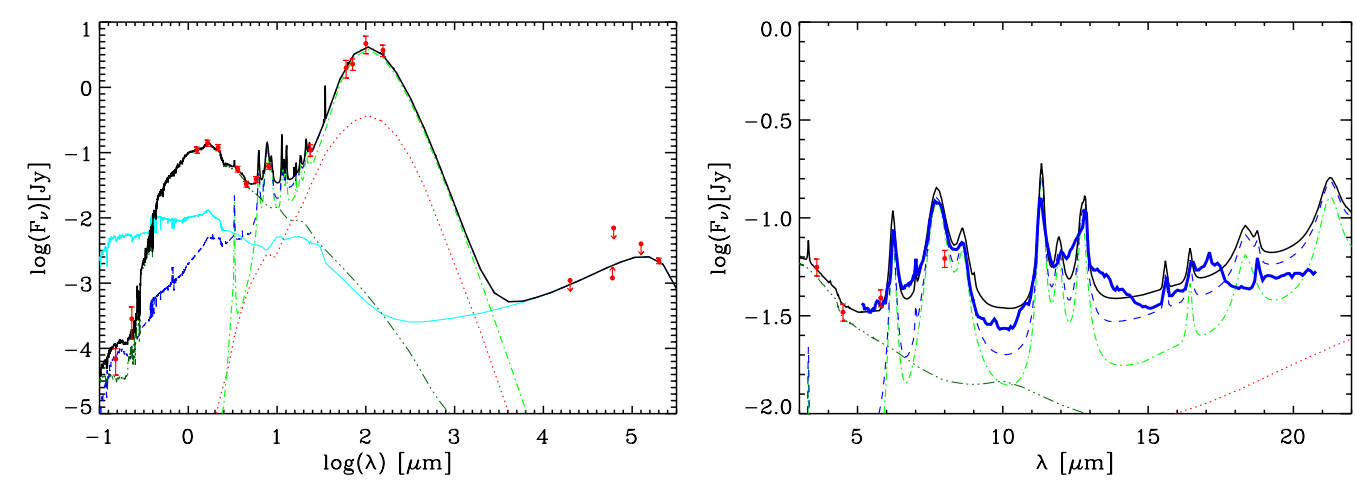

Figure 1. Comparison between the observed SED of the central region of NGC 4435 and the GRASIL model. The thick solid line represents the model for the total SED, i.e. the starburst component plus the old stellar component; the three dots-dashed dark green line represents the contribution from the old stellar population, and the dashed blue line represents the total contribution from the burst of star formation, the dotted red line represents the emission from molecular clouds, the dot-dashed green line represents the diffuse medium emission and the thin solid cyan line denotes the emission from stars of the starburst component without applying the extinction from dust. The filled red circles are the broad band data. Left: Comparison from $0.1 \mu \mathrm{m}$ to $100 \mathrm{MHz}$. Right: Comparison for the MIR wavelengths. The thickest solid blue line represents the IRS Spitzer spectrum.

\section{Early-type galaxies in the mid infrared.}

Eighteen ETGs among those that define the colour-magnitude relation of the Virgo cluster (Bower, Lucy \& Ellis 1992) were observed in standard staring mode with the low resolution IRS modules between 5 and $20 \mu \mathrm{m}$, in January and July 2005. The calibration and spectra extraction procedures are discussed in detail in Bressan et al. (2006a). The spectra of these galaxies are shown in Bressan et al. (2006a) and Bressan et al. (2007).

For thirteen galaxies $(76 \%)$ of our sample, the MIR spectrum is characterized by the presence of a broad emission features above $10 \mu \mathrm{m}$, without any other narrow emission feature. The analysis of the IRS spectra indicates that the $10 \mu \mathrm{m}$ feature has an extended spatial distribution; moreover its spatial distribution is consistent with that obtained below $8 \mu \mathrm{m}$, where the spectra are dominated by stellar photospheres. This result has been confirmed by the analysis of Spitzer IRS Peak-Up imaging observations in the blue $(16 \mu \mathrm{m})$ filter of selected galaxies (Annibali et al. in preparation). It is also in agreement with previous ISOCAM observations that indicated spatially resolved emission at both 6.7 and $15 \mu \mathrm{m}$ (Athey et al. 2002, Ferrari et al 2002, Xilouris et al. 2004). In view of these considerations and based on preliminary fits with our models of passively evolving old simple stellar populations, we argued that we have detected the $10 \mu \mathrm{m}$ features, due to silicate emission from the circumstellar envelopes of mass losing AGB stars, as predicted by Bressan et al. (1998). Bressan et al. (2007) have recently shown that the $10 \mu \mathrm{m}$ feature observed in early type galaxies is similar in shape but about a factor four larger than the semi empirical one obtained for the globular cluster 47 Tuc, consistent with a metallicity variation of the same order. We are now computing new isochrones and SSP models that account for a more realistic description of the AGB phase and of their dusty envelopes.

Among bright Virgo cluster ETGs observed by our team, four galaxies (24\%) show various levels of activity. NGC 4636 (optically classified as a LINER) shows low ionization emission lines ([ArII $] 7 \mu \mathrm{m},[\mathrm{NeII}] 12.8 \mu \mathrm{m},[\mathrm{NeIII}] 15.5 \mu \mathrm{m}$ and $[\mathrm{SIII}] 18.7 \mu \mathrm{m})$ on a continuum similar to other passive galaxies. NGC 4486 (M87) shows the same emission lines on a continuum dominated by the AGN emission above $8 \mu \mathrm{m}$. The broad continuum 
feature above $10 \mu \mathrm{m}$ in M87 could be caused by silicate emission from the dusty torus (Siebenmorgen et al. 2005, Hao et al. 2005). NGC 4550 shows some PAH emission features while the MIR SED of NGC 4435 is characteristic of a star forming object.

\section{The panchromatic SED of NGC 4435}

NGC 4435 is an S0 galaxy interacting with NGC 4438 and it hosts a circumnuclear disk. Panuzzo et al. (2007) combined the Spitzer IRS spectra of NGC 4435 with IRAC and MIPS archival data and existing broad band measurements from X-ray to radio wavelengths to obtain an accurate panchromatic spectral energy distribution (SED) of this galaxy. The SED was analysed with GRASIL (Silva et al. 1998) and well reproduced at all wavelengths. The analysis shows that the circumnuclear disk experienced a burst of star formation activity which is now fading.

The IRS data themselves provide precise answers on important questions such as the nature of the nuclear activity suspected from optical (Ho et al. 1997) and X-ray (Machacek et al. 2004) observations, and the metallicity of the gas in the circumnuclear disk. We fail to detect any high excitation nebular emission lines in the IRS spectrum; the $[\mathrm{NeIII}] 15.5 /[\mathrm{NeII}] 12.8$ ratio constrains the contribution of a possible AGN to the ionizing flux to be less than $2 \%$. The upper limit on the temperature derived from $\mathrm{H}_{2} \mathrm{~S}(1)$ and $\mathrm{S}(2)$ rotational lines is lower than expected for AGN excitation and PAH features are well reproduced by star formation models. Moreover, the X-ray emission is within the range expected from X-ray binaries in an advanced phase of the starburst.

As for the metallicity of the nuclear disk, the comparison of observed MIR nebular lines with those predicted by the GRASIL model (Panuzzo et al 2003) indicates that it is almost solar. This is one of the first accurate direct estimates of the gas metallicity in ETGs.

The age of the starburst, $\sim 180 \mathrm{Myr}$, corresponds to the epoch of the onset of the interaction with NGC 4438 derived from dynamical simulations (Combes et al. 1988). The mass of stars born during the starburst $\left(\sim 1.22 \times 10^{8} M_{\odot}\right)$ amounts to about $1.5 \%$ of the stellar mass sampled by the central 5 arcsec aperture.

\section{Conclusions}

We have obtained with Spitzer IRS mid infrared spectra of ETGs selected along the colour-magnitude relation of the Virgo cluster.

The mid infrared SED of most of our ETGs shows a clear broad emission around $10 \mu \mathrm{m}$ and longward as predicted in Bressan et al. (1998) which is likely due to dusty mass losing AGB stars. In the remaining fraction of galaxies (24\%) we detect signatures of activity at various levels. The analysis of the IRS spectrum of NGC 4435 testifies to the superb capability of Spitzer to probe the nature of this type of activity and supports the notion that ETGs with relatively strong hydrogen absorption features are due to recent small rejuvenation episodes, rather than being the result of delayed galaxy formation (Bressan et al. 1996).

\section{Acknowledgements}

A. B., G.L. G. and L. S. thank INAOE for warm hospitality.

\section{References}

Athey, A., Bregman, J., Bregman, J., Temi, P., \& Sauvage, M. 2002, ApJ, 571, 272 
Bower, R. G., Lucey, J. R., Ellis, R. S. 1992, MNRAS, 254, 601

Bressan, A., et al. 2006, ArXiv Astrophysics e-prints, arXiv:astro-ph/0701620

Bressan, A., et al. 2006, ApJl, 639, L55

Bressan, A., et al. 2006, ArXiv Astrophysics e-prints, arXiv:astro-ph/0604068

Bressan, A., Granato, G.L., \& Silva, L. 1998, A\&A, 332, 135

Bressan, A., Chiosi, C., \& Tantalo, R. 1996, A\&A, 311, 425

Combes, F., Dupraz, C., Casoli, F., \& Pagani, L. 1988, A\&A, 203, 9

Ferrari, F., Pastoriza, M. G., Macchetto, F. D. et al. 2002, A\&A, 389, 355

Hao, L., et al. 2005, ApJl, 625, L75

Ho, L. C., Filippenko, A. V., \& Sargent, W. L. 1997, ApJS, 112, 315

Houck, J.R. 2004, ApJS, 154, 18

Kaneda, H., Onaka, T., \& Sakon, I. 2005, ApJ, 632, L83

Machacek, M. E., Jones, C., \& Forman, W. R. 2004, ApJ, 610, 183

Panuzzo, P., et al. 2007, ApJ in press (astro-ph/0610316)

Panuzzo, P., Bressan, A., Granato, G. L., Silva, L., \& Danese, L. 2003, A\&A, 409, 99

Siebenmorgen, R., Haas, M., Krügel, E., \& Schulz, B. 2005, A\&A, 436, L5

Silva, L., Granato, G. L., Bressan, A., \& Danese, L. 1998, ApJ, 509, 103

Xilouris, E. M. et al. 2004, A\&A, 416, 41

\section{Discussion}

MEIXNER: I'm happy to see that someone is including dust in their models. You say that the $10 \mu \mathrm{m}$ emission in the elliptical is extended. Is your interpretation that the AGB population is extended? Is the extended emission consistent with the AGB population as traced by near-IR photometry?

BRESSAN: Our IRS data analysis pipeline allows an accurate check of the spatial distribution of the emission. In the passive galaxies, the emission looks extended at all wavelengths. The spatial extension is consistent with IRAC and 2MASS images. The preliminary analysis of our Spitzer IRS Peak-Up imaging observations confirms the extension also at $16 \mu \mathrm{m}$. This indicates that the $10 \mu \mathrm{m}$ excess is of stellar origin, likely from the extended population of AGB stars.

RENZINI: One may expect that dust particled from AGB stars are destroyed by sputtering due to interaction with the hot, X-Ray emitting ISM of these galaxies. Is there any correlation of the strength of the emission with the X-ray luminosity of these galaxies?

BRESSAN: We did not compare with the X-ray luminosity yet. However the dust distribution from stationary circumstellar envelopes is proportional to $\mathrm{r}^{-2}$ and the emission is dominated by the innermost layers, where dust is likely not affected by interaction with the hot ISM. 